\title{
Exploring Forces between Individual Colloidal Particles with the Atomic Force Microscope
}

\author{
Prashant Sinha§, lonel Popa, Marco Finessi, Francisco J. M. Ruiz-Cabello, István Szilágyi, \\ Plinio Maroni, and Michal Borkovec*
}

§SCS-Metrohm Foundation Award for best oral presentation

\begin{abstract}
Forces between individual colloidal particles can be measured with the atomic force microscope (AFM), and this technique permits the study of interactions between surfaces across aqueous solutions in great detail. The most relevant forces are described by the Derjaguin, Landau, Verwey, and Overbeek (DLVO) theory, and they include electrostatic double-layer and van der Waals forces. In symmetric systems, the electrostatic forces are repulsive and depend strongly on the type and concentration of the salts present, while van der Waals forces are always attractive. In asymmetric systems, the electrostatic force can become attractive as well, even when involving neutral surfaces, while in rare situations van der Waals forces can become repulsive too. The enormous sensitivity of the double layer forces on additives present is illustrated with oppositely charged polyelectrolytes, which may induce attractions or repulsions depending on their concentrations.
\end{abstract}

Keywords: Atomic force microscope $\cdot$ Colloidal probe technique $\cdot$ DLVO theory $\cdot$ Double-layer forces · van der Waals forces

\section{Introduction}

Many phenomena involving colloidal particles, which typically are in a size range between nanometers and micrometers, are governed by mutual interaction forces. Water treatment plants rely on attractive forces between suspended particles to induce formation of large aggregates that can be separated by sedimentation. ${ }^{[1]}$ Papermaking equally requires attractive forces between cellulose fibers and filler particles in order to rapidly form large aggregates in the paper machine. ${ }^{[2]}$ Repulsive forces are essential to maintain stable and easily flowing suspensions of colloidal

${ }^{\star}$ Correspondence: Prof. Dr. M. Borkovec Department of Inorganic, Analytical and Applied Chemistry

Sciences II

30, Quai Ernest-Ansermet

$\mathrm{CH}-1211$ Geneva 4

Tel.: +4122 3796405

Fax: +41223796069

E-mail: michal.borkovec@unige.ch particles even at high solid concentrations, which are important in paints, foods, or cosmetics. ${ }^{[3,4]}$

The most relevant forces acting between colloidal particles across aqueous solutions can be often rationalized within the classical theory from Derjaguin, Landau, Verwey, and Overbeek (DLVO). ${ }^{[5-8]}$ This theory stipulates that interaction forces can be approximated by the sum of electrostatic double-layer repulsion and van der Waals dispersion interactions. The double-layer repulsion originates from the buildup of an osmotic pressure between two overlapping diffuse layers. Such forces only occur when at least one of the surfaces is charged, and they sensitively depend on the presence of charged chemical species. The van der Waals interaction, which is also referred to as the Casimir force, originates from the dispersion attraction between fluctuating permanent or induced dipoles, and is mostly attractive. In some situations, non-DLVO forces may become important, and they may include interactions due to inhomogeneous charge distributions, ${ }^{[9]}$ adsorbed polymer layers, ${ }^{[10]}$ or depletion forces induced by suspended particles or polymers. ${ }^{[11]}$ Nevertheless, the simple DLVO picture is capable of rationalizing interaction forces in a wide variety of systems, and illustrative examples will be discussed in the present article.

For a long time, only indirect methods were available to address interaction forces between colloidal particles (e.g. osmometry, scattering). The idea to measure the respective forces directly evolved from Derjaguin's force balances used to measure forces between macroscopic objects ${ }^{[12]}$ and the subsequently developed surface forces apparatus. ${ }^{[8]}$ The latter apparatus did provide first reliable measurements of DLVO forces between curved mica sheets. The necessary miniaturization of the force balance was made possible through the invention of the atomic force microscope (AFM) by Binnig and coworkers. ${ }^{[13]}$ First direct force measurements involving colloidal particles with the AFM were independently realized by Ducker and Butt.[14,15] While other techniques permitting force measurements involving individual colloidal particles have been developed in the meantime, such as total internal reflection microscopy (TIRM) ${ }^{[16]}$ or optical tweezers techniques, ${ }^{[17]}$ it is probably fair to state that the colloidal probe technique is currently the most promising one, and is being used in numerous laboratories worldwide. ${ }^{[9-11,18-26]}$

\section{Colloidal Probe Technique}

The key idea is to replace the sharp AFM-tip with a colloidal particle with a diameter of a few $\mu \mathrm{m}$ (see Fig. 1a). This particle is normally glued to the cantilever in the 
dry state with a micromanipulator. ${ }^{[14,15,21]}$ Alternative in situ techniques have been developed permitting the attachment of particles in solution without a drying-rewetting step. ${ }^{[9,20]}$ With a standard AFM one approaches the colloidal probe vertically to the substrate and measures the cantilever deflection by means of a reflected light beam. As the force constant of the cantilever can be measured independently, for example through its thermal fluctuations, the deflection can be converted to the interaction force. Typical force resolution achievable with colloidal probe AFM are few tens of $\mathrm{pN}$, but the resolution can be improved by subsequent averaging of individual force profiles. While the separation distance can also be measured with TIRM independently, ${ }^{[27]}$ one normally obtains the distance from the contact point between the probe and the substrate (i.e. onset of constant compliance). For solid substrates, the latter technique yields separation distances with an accuracy better than nanometers.

With this setup one can measure the force between a colloidal particle and flat substrate (asymmetric system, Fig. 1a). ${ }^{[14,15,27]}$ Attaching another particle to the substrate, one can measure the interaction between two colloidal particles (symmetric system, Fig. 1b). ${ }^{[20,21]}$ In this case, the particles have to be centered laterally prior to the measurement, which can be achieved with an AFM mounted on an inverted optical microscope. By attaching one type of particles to the tip and another type to the substrate, the interaction between two different particles can be investigated (asymmetric system, Fig. 1c). ${ }^{[20]}$

\section{Double Layer Repulsion}

When particles are highly charged, interaction forces are dominated by the electrostatic double-layer force. Such forces act between two positively charged amidine latex particles at different salt concentrations, and they are shown in Fig. 2. One observes that they strongly depend on the ionic strength. For larger separations, this force is exponential, which leads to a straight line in the semi-logarithmic representation used. ${ }^{[8]}$ Its slope reflects the thickness of the diffuse layer, which is referred to as the Debye length. This length is inversely proportional to the square root of the ionic strength. The intercept is related to the electrical surface potential or the surface charge density. From an experiment involving two similar particles (i.e. symmetric system) one can only find the magnitude of the charge density unambiguously, but the sign of the potential cannot be determined since two equally charged surfaces always repel. This sign must be obtained from force measurements in asymmetric systems or another technique (e.g. electrophoresis). The solid lines shown in Fig. 2 are fits obtained from the solution of the full Poisson-Boltzmann equation. From these calculations, one can extract a surface charge density of $3.5 \mathrm{mC} /$ $\mathrm{m}^{2}$. This charge density is very similar to the value obtained by electrophoresis, but its magnitude is substantially smaller than the one expected from conductometric data. The latter discrepancy indicates that the surface charge is partially neutralized by strongly bound counter ions.

When two charged surfaces approach, they will have the tendency to decrease the magnitude of their surface charge density in order to reduce the overall free energy. This decrease may result from the dissociation of surface groups as well as the ion adsorption or desorption from the surface. This aspect can be considered quantitatively by considering the appropriate boundary conditions. For constant charge (CC) conditions, the charge will remain constant upon approach, while for constant potential (CP) conditions, the potential will remain constant but the surface charge will be reduced upon approach. The constant regulation (CR) conditions describe the

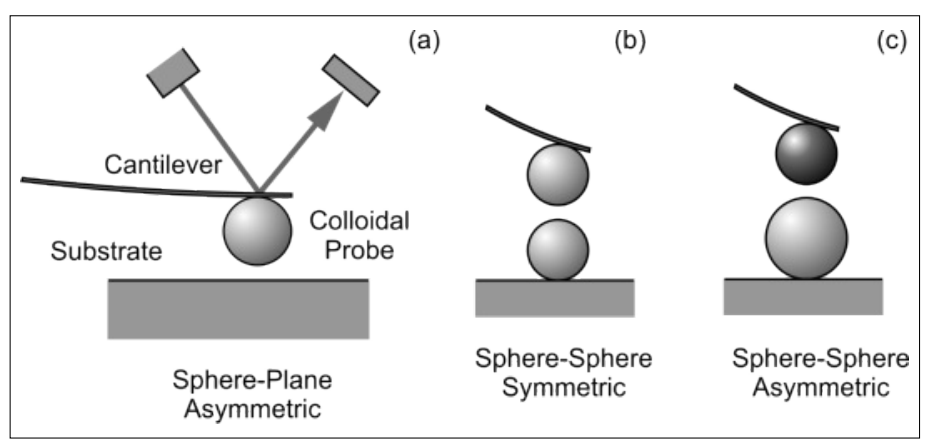

Fig. 1. Colloidal probe atomic force microscopy (AFM). Force measurement between (a) particle and planar substrate, (b) two similar colloidal particles, and (c) two dissimilar colloidal particles.

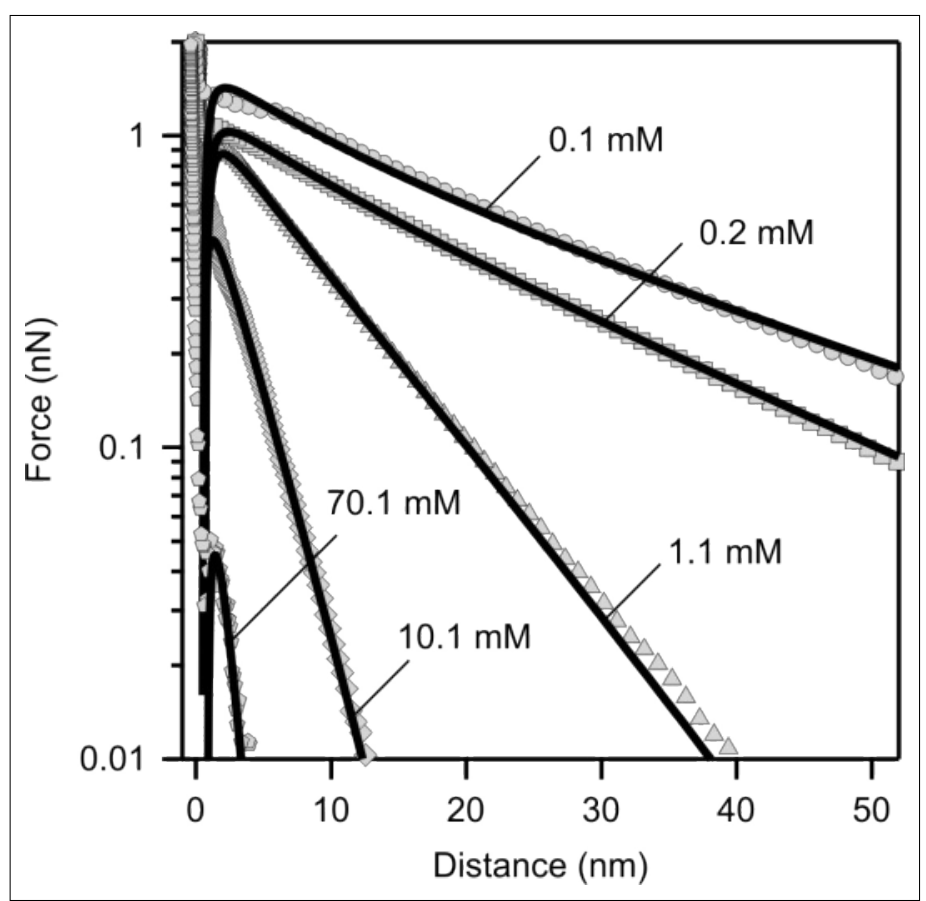

Fig. 2. Force profiles between two amidine latex particles at $\mathrm{pH} 4.0$ across $\mathrm{KCl}$ electrolyte solutions of varying ionic strengths. Solid lines are best fits with DLVO theory. 

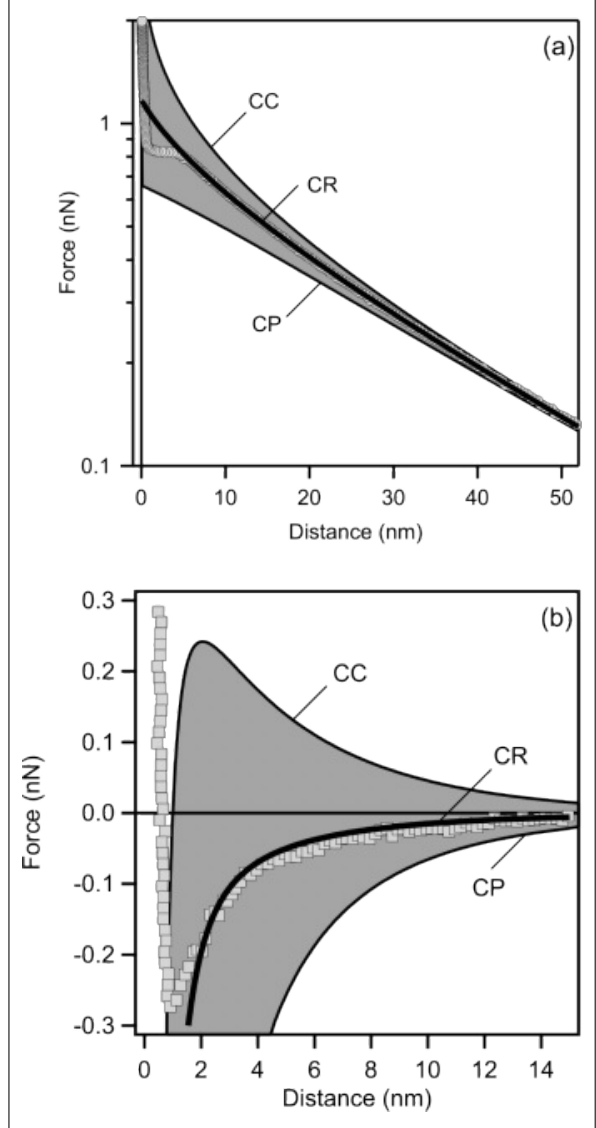

Fig. 3. Effect of charge regulation on electrostatic double layer-interactions with constant charge (CC), constant regulation (CR), and constant potential (CP) boundary conditions. (a) Symmetric system with two sulfate latex particles at $\mathrm{pH} 4.0$ with best fits with the electrostatic double-layer force. (b) Asymmetric system comprising a neutral amidine and negatively charged sulfate latex particle at $\mathrm{pH}$ 5.6 with predictions of DLVO theory. Note the larger effect of charge regulation in (b).

diameter of $3.1 \mu \mathrm{m}$ are reported at an ionic strength of $0.2 \mathrm{mM}$. The surface charge density of the sulfate particles is $-2.1 \mathrm{mC} / \mathrm{m}^{2}$. In this case, $\mathrm{CC}$ conditions lead to a repulsive double layer force, while CP conditions to an attractive one. In reality, the observed forces are somewhat attractive, suggesting that the regulation behavior resembles CP rather than CC. The solid line is a prediction of the Poisson-Boltzmann model with regulation parameters of 0.41 for the amidine latex and 0.33 for the sulfate latex. All input parameters of this calculation were obtained from force measurements in the two respective symmetric systems. This model is capable of predicting the force profiles in the asymmetric system very well.

\section{Van der Waals Attraction}

Colloidal particles always interact by attractive van der Waals forces. These forces can be measured most easily when the particles are neutral. Fig. 4 shows such measurements between two neutral amidine particles of $3.3 \mu \mathrm{m}$ in diameter. The solid line represents the fit with the expected van der Waals force, which follows an inverse dependence on the square of the separation distance at sufficiently small distances. ${ }^{[7,8]}$ The strength of this force is characterized by the Hamaker constant, and one obtains the value of $4.5 \times 10^{-21} \mathrm{~J}$. Independent calculations based on Lifshitz theory suggest a somewhat larger value of $9.0 \times 10^{-21} \mathrm{~J}$. We suspect that the observed discrepancy is probably due to surface roughness effects. The attractive force is much more difficult to observe when the particles are charged. The reason for these difficulties is that the extent of the attractive region is very small and difficult to detect due to surface roughness or a mechanical instability of the cantilever. In symmetric systems, van der Waals forces are always attractive. In asymmetric systems, van der Waals forces may sometimes become repulsive. ${ }^{[8,29]}$ One important example is a gas bubble interacting with a mineral surface across water, but this case will not be discussed here.

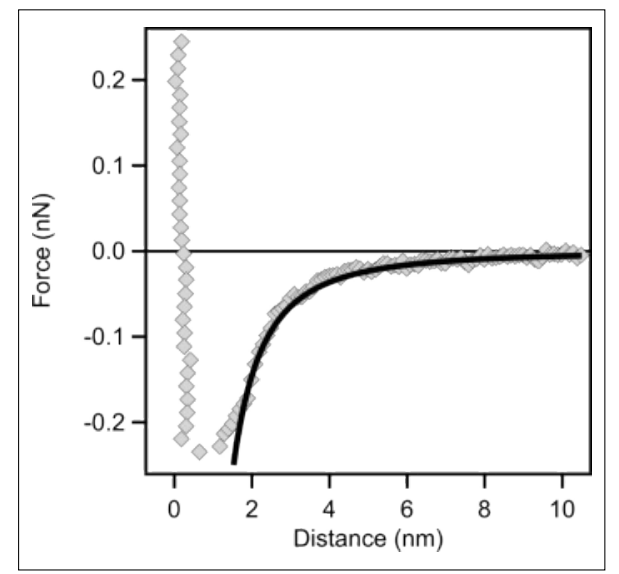

Fig. 4. Attractive van der Waals force between two neutral amidine latex particles at pH 5.6. Solid line is the best fit with the van der Waals attraction.

\section{Charge Reversal by Polyelectrolytes}

As the last example, let us illustrate how strongly interaction forces between colloidal particles can be modified by adsorption of charged species. Such effects are very pronounced upon adsorption of oppositely charged polyelectrolytes. ${ }^{[24]}$ The system to be discussed involves negatively charged sulfate latex particles of diameter of 3.1 $\mu \mathrm{m}$ at an ionic strength of $1.1 \mathrm{mM}$ in the presence of linear poly(ethylene imine) (LPEI). This cationic weak polyelectrolyte is partially charged under the conditions investigated and adsorbs strongly to

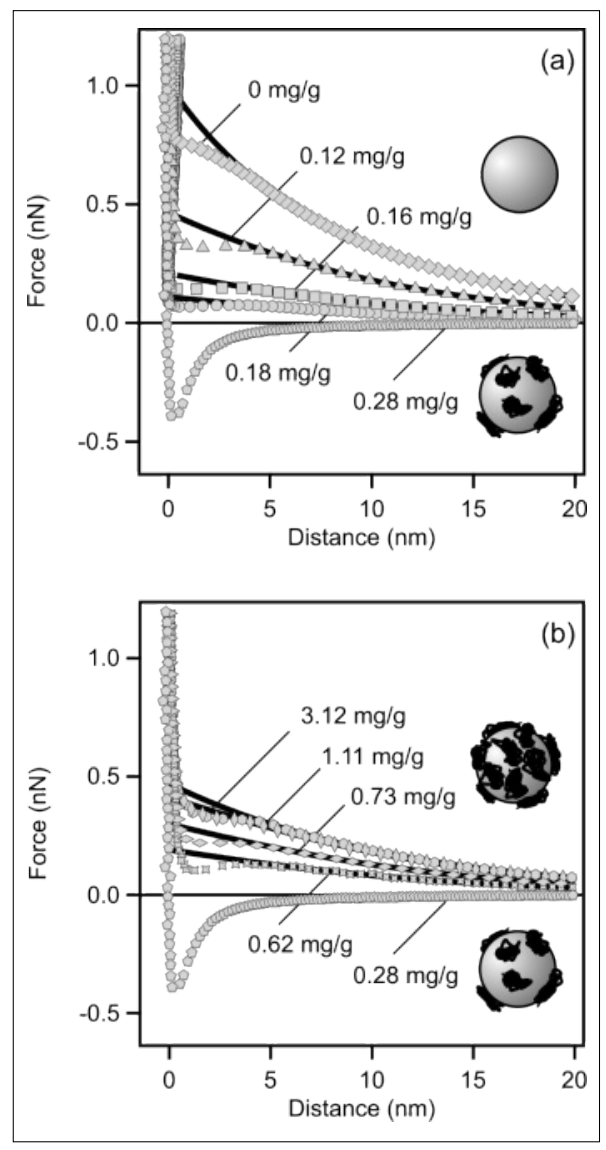

Fig. 5. Force profiles between sulfate latex particles for various doses of linear poly(ethylene imine) (LPEI) at an ionic strength of $1.1 \mathrm{mM}$ and $\mathrm{pH}$ 4.0. (a) Below and at the charge neutralization point, and (b) at this point and above. Solid lines are the best fits with electrostatic double layer force.

the particle surface. The force profiles in this system are shown in Fig. 5. The bare particles show repulsive forces dominated by double layer repulsion. When LPEI is added to the system, the surface charge is reduced, since the adsorbed LPEI partially neutralizes the particle charge. As a consequence, the repulsive forces are weakened (Fig. 5a). At a particular dose, the surface charge is precisely neutralized, and only the attractive van der Waals force can be observed. When the dose is increased further, the particles become positively charged due to an excess of adsorbed LPEI (Fig. 5b).

This charge reversal is probably caused by ion-correlation effects or additional non-electrostatic interactions acting between LPEI and the particle. Under these conditions, the particles are repulsive again due to double layer forces. The surface charge continues to increase until the adsorbed amount saturates, and beyond this point the repulsive force remains constant. Indeed, by varying the dose of LPEI, the interaction forces can be widely tuned, namely from repulsive to attractive and again back to repulsive. Other polyelectrolyte-particle systems feature stronger 
attractive forces, which originate from the laterally heterogeneous charge distribution in the adsorbed polyelectrolyte films. ${ }^{[9]}$

\section{Conclusion}

This article summarizes the capability of the AFM-based colloidal probe technique to directly measure interaction forces between individual colloidal particles. Highly charged particles interact through repulsive double layer forces. The range of these forces is characterized by the ionic strength of the solution and their magnitude by the surface charge density (or surface potential). Attractive van der Waals forces can be investigated by force measurements between neutral particles. Strongly adsorbing charged species modify the interaction forces substantially as illustrated with oppositely charged polyelectrolytes. Adsorbing polyelectrolytes may completely neutralize the surface charge but upon further addition lead to charge reversal. In such systems, the forces may even vary from repulsive through attractive and back to repulsive.

\section{Acknowledgement}

This work was supported by the Swiss National Science Foundation and the University of Geneva.

Received: January 16, 2012

[1] B. Bolto, J. Gregory, Water Res. 2007, 41, 2301.

[2] J. Porubska, B. Alince, T. G. M. van de Ven, Colloid Surf. A 2002, 210, 223.

[3] P. G. de Gennes, Nature 2001, 412, 385.

[4] A. R. Studart, E. Amstad, L. J. Gauckler, Langmuir 2007, 23, 1081.

[5] B. Derjaguin, L. D. Landau, Acta Phys. Chim. 1941, 14, 633.

[6] E. J. W. Verwey, J. T. G. Overbeek, 'Theory of Stability of Lyophobic Colloids', Elsevier, Amsterdam, 1948.

[7] W. B. Russel, D. A. Saville, W. R. Schowalter, 'Colloidal Dispersions', Cambridge University Press, Cambridge, 1989.

[8] J. Israelachvili, 'Intermolecular and Surface Forces', Academic Press, London, 1992.

[9] I. Popa, G. Gillies, G. Papastavrou, M. Borkovec, J. Phys. Chem. B 2010, 114, 3170.

[10] S. Block, C. A. Helm, Phys. Rev. E 2007, 76, 030801.

[11] N. C. Christov, K. D. Danov, Y. Zeng, P. A. Kralchevsky, R. von Klitzing, Langmuir 2010, 26, 915.

[12] B. V. Derjaguin, Y. I. Rabinovich, N. V. Churaev, Nature 1978, 272, 313

[13] G. Binnig, C. F. Quate, C. Gerber, Phys. Rev. Lett. 1986, 56, 930 .
[14] W. A. Ducker, T. J. Senden, R. M. Pashley, Nature 1991, 353, 239.

[15] H. J. Butt, Biophys. J. 1991, 60, 1438.

[16] D. C. Prieve, Adv. Colloid Interface Sci. 1999 82, 93 .

[17] J. Baumgartl, J. L. Arauz-Lara, C. Bechinger, Soft Matter 2006, 2, 631

[18] H. J. Butt, B. Cappella, M. Kappl, Surf. Sci. Rep. 2005, 59, 1.

[19] P. M. Claesson, T. Ederth, V. Bergeron, M. W. Rutland, Adv. Colloid Interface Sci. 1996, 67, 119.

[20] I. Popa, M. Finessi, P. Sinha, P. Maroni, G Papastavrou, M. Borkovec, Phys. Rev. Lett. 2010, 104, 228301.

[21] G. Toikka, R. A. Hayes, J. Ralston, Langmuir 1996, 12, 3783.

[22] V. Kane, P. Mulvaney, Langmuir 1998, 14, 3303.

[23] D. Qu, D. Baigl, C. E. Williams, H. Möhwald, A. Fery, Macromolecules 2003, 36, 6878 .

[24] M. Finessi, P. Sinha, I. Szilagyi, I. Popa, P. Maroni, M. Borkovec, J. Phys. Chem. B 2011 115, 9098.

[25] M. Dishon, O. Zohar, U. Sivan, Langmuir 2009 25, 2831.

[26] S. Pasche, M. Textor, L. Meagher, N. D. Spencer, H. J. Griesser, Langmuir 2005, 21, 6508.

[27] S. C. Clark, J. Y. Walz, W. A. Ducker, Langmuir 2004, 20, 7616.

[28] M. Borkovec, S. H. Behrens, J. Phys. Chem. B 2008, 112, 10795.

[29] A. Milling, P. Mulvaney, I. Larson, J. Colloid Interface Sci. 1996, 180, 460 